\title{
Evaluation of the Effects of Hypo-Magnetic Fields on Mouse Macrophage RAW264 Cells
}

\author{
Nipitpon Srimai ${ }^{1,2}$, Wasawat Kiatarkom ${ }^{1,2}$, Hidetake Miyata $^{2}$ \\ ${ }^{1}$ Department of Biology, Faculty of Science, Naresuan University, 99 Moo 9 Phitsanulok-Nakhonsawan Road Tambon \\ Tapho, Muang, Phitsanulok, 65000, Thailand \\ ${ }^{2}$ Department of Physics, Faculty of Science, Tohoku University, Aza-Aoba 6-3, Aramaki, Aoba-ku, Sendai, Miyagi \\ 980-8578, Japan
}

Correspondences: Hidetake Miyata, Department of Physics, Faculty of Science, Tohoku University, Miyagi 980-8578, Japan.E-mail: miyata@bio.phys.tohoku.ac.jp

Received: September 15, 2020 Accepted: November 30, 2020 Online Published: December 20, 2020

doi:10.5539/ijc.v13n1p12

URL: https://doi.org/10.5539/ijc.v13n1p12

\begin{abstract}
Hypo-magnetic fields (HMFs) are static magnetic fields that are significantly weaker than the geomagnetic field and are found in interplanetary space, on Mars, and the Moon. Understanding the mechanism of the biological effects of HMFs is an important topic in magnetobiology. Human physiology studies have shown that HMFs cause various adverse health effects such as increased blood pressure and blood flow rate. Previous studies on the effects of HMFs on cells revealed a variety of effects, such as a change in growth rate, a decrease in mitochondrial membrane potential, and an increase in reactive oxygen species. A decrease in mitochondrial membrane potential $\left(\Delta \Phi_{\mathrm{m}}\right)$ may seriously impair cellular activity by interfering with the production of ATP. Thus, a deeper investigation of the effects of HMFs on mitochondrial function could provide clues to understanding the adverse effects of HMFs. In the present study, we used mouse macrophage RAW264 cells to investigate the effects of an HMF on mitochondrial membrane potential, mitochondrial superoxide anion $\left(\mathrm{O}_{2}^{-}\right)$production, and cell proliferation.
\end{abstract}

Keywords: geomagnetic field, mitochondrial membrane potential, superoxide anion, cell proliferation

\section{Introduction}

Magnetic fields in inter-planetary space and on Mars and the Moon are at least ten times weaker than Earth's geomagnetic field (GMF) (Kokhan et al., 2016). Such weak fields are often called hypo-magnetic fields (HMFs). Several physiological effects of HMFs are known. For example, they affect cardiovascular functions such as capillary blood pressure, blood circulation, and the duration of cardio intervals (Gurfinkel et al., 2016). Immune cells isolated from rats kept under HMF conditions $(<12 \mu \mathrm{T})$ for 6 months showed decreased release of nitric oxide and decreased synthesis of $\mathrm{O}_{2}^{-}$(Roman and Tombarkiewicz, 2009), raising the concern that HMFs may adversely affect human health during long space missions.

Cellular level studies are thus required to understand the mechanism of the health effects of HMFs. Previous studies have shown that an HMF $(<0.5 \mu \mathrm{T})$ decreased the proliferation/growth of model cancer cells (Martino et al., 2010) and stimulated the proliferation/cell division of mouse neural progenitor and stem cells (Fu et al., 2016a). Another study showed that a $<3 \mu \mathrm{T}$ HMF reduced the viability of skeletal muscle cells and was accompanied by a decline in mitochondrial functions (e.g., $\Delta \Phi_{\mathrm{m}}$; Fu et al., 2016b).

Mitochondria may be the most sensitive of cellular organelles to reduced magnetic fields and could affect their structure and function (Belyavskaya, 2001, 2004). Furthermore, a 50-Hz square-wave magnetic field was found to promote human sperm motility (Iorio et al., 2011), and a 50-Hz sinusoidal magnetic field altered the structure of cristae in chicken embryo heart mitochondria (Lahijani et al., 2013) and lowered $\Delta \Phi_{\mathrm{m}}$ (Pooam et al., 2017). Thus, mitochondria may be key to understanding the biological effects of magnetic fields. Since the mitochondrion is the ATP-producing organelle, investigating how HMFs cause a decrease in mitochondrial function may provide clues to understanding the mechanism of the biological action of HMFs.

Here, we used mouse macrophage RAW264 cells to investigate the effects of HMFs on $\Delta \Phi_{\mathrm{m}}, \mathrm{O}_{2}{ }^{-}$production in mitochondria and cytoplasm, and on cell proliferation. We found that under the HMF conditions tested, the mitochondrial membrane potential decreased, cell proliferation decreased, but the $\mathrm{O}_{2}^{-}$level was not affected. These 
results appear difficult to reconcile with our current understanding of HMFs as inhibitors of mitochondrial function.

\section{Materials and Methods}

\subsection{Cells and Reagents}

The RAW264 macrophage cell line cell was obtained from the RIKEN BioResource Center (Wako, Saitama, Japan). Minimum essential medium (MEM) with phenol red plus non-essential amino acids, MEM without phenol red and non-essential amino acids, fetal bovine serum (FBS), and penicillin-streptomycin were obtained from Gibco $^{\odot}$ Life Technologies (Tokyo, Japan). 3,3'-[3,3'-Dimethoxy-(1,1'-biphenyl)-4,4'-diyl]-bis[2-(4-nitrophenyl)-5-phenyl-2H-tetrazolium chloride] (nitro-TB) was obtained from Dojindo Laboratories (Kumamoto, Japan). MitoSox ${ }^{\mathrm{TM}}$ Red and 5,5',6,6'-tetrachloro-1,1',3,3'-tetraethylbenzimidazolylcarbocyanine iodide (JC-1) were obtained from Thermo Fisher Scientific (Rockford, IL, USA). 2'-(4-Ethoxyphenyl)-5-(4-methyl-1-piperazinyl)-2,5-bi-1H-benzimidazole trihydrochloride (Hoechst 33342) and 3,8-diamino-5-[3-(diethylmethylammonio)propyl]-6-phenylphenanthridinium diiodide (propidium iodide) were obtained from Immunochemistry Technologies LLC (Bloomington, MN, USA). A luciferin-luciferase-based ATP assay kit was obtained from Toyo B-Net (Tokyo, Japan). Bovine serum albumin was from Sigma (Tokyo, Japan).

\subsection{Cell Exposure System}

Figure 1A shows the shielding case made of permalloy (Ohtama Co., Ltd., Tokyo, Japan) and Figure 1B shows the $\mathrm{CO}_{2}$ incubator used (Model IT-600; Yamato Scientific Co., Tokyo, Japan) placed in the shielding case. Figure 1C shows the custom-made (Hozen Industries Co., Ltd., Kyoto, Japan) Merritt coil system (Merritt et al., 1983) placed in the incubator. Figure 1D shows how the culture dishes were placed in the Merritt coil system. We used two identical set-ups. The Merritt coil system consisted of four sub-coils. Each coil was constructed by winding two parallel electrical wires around a square aluminum frame $(20 \mathrm{~cm} \times 20 \mathrm{~cm} \times 3.5 \mathrm{~cm})$. The electric currents in the two wires ran in the same direction in the exposure mode, and in opposite directions in "active-sham" mode. The coil current was generated using a function generator (model WF1973, NF Corporation, Yokohama, Japan).

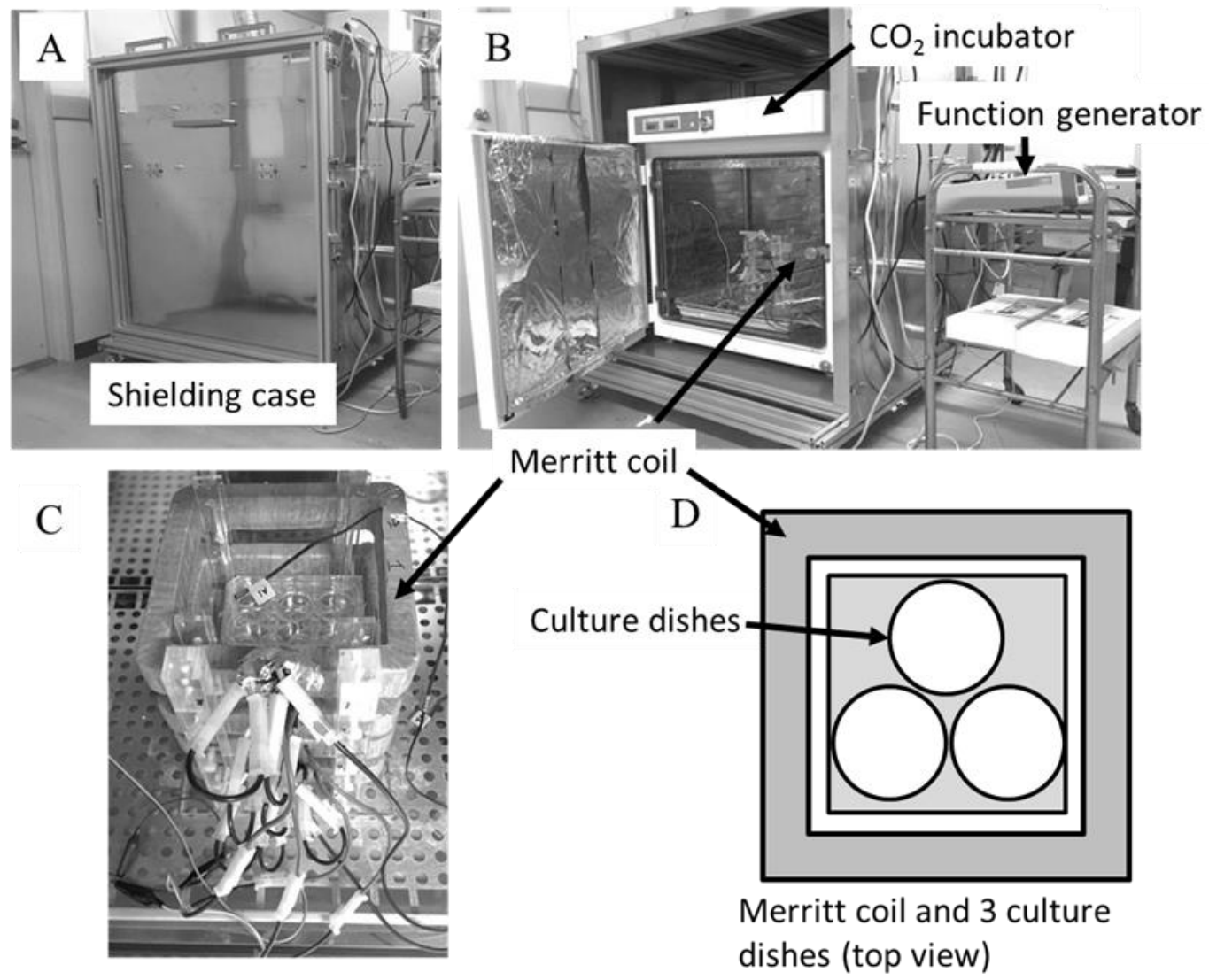

Figure 1. A) The magnetic shielding case, $\mathrm{B}$ ) the $\mathrm{CO}_{2}$ incubator placed in the shielding case, and the function generator, C) a close-up of the Merritt coil system, D) a schematic representation of the 3 culture dishes placed on a support (light grey) in the Merritt coil system. The coil current was generated using a function generator and was provided to the coil through a 4.8-ohm resistor 
The magnetic flux density in the vertical direction $\left(B_{\mathrm{V}}\right)$ was measured with a Gauss meter (Model 475 DSP; Lake Shore, Westville, OH, USA) equipped with an axial-type Hall-effect probe (HMMA-2504-VR; Lake Shore), and that in the horizontal plane $\left(B_{\mathrm{H}}\right)$ was measured with a Gauss meter (Model 421; Lake Shore) equipped with a transverse-type Hall-effect probe (MMT-4E04-VH; Lake Shore). In the exposure mode, the coil current (provided through a $4.8 \Omega$ resistor) was adjusted to $\sim 0.1$ A to achieve $B_{\mathrm{V}}=-46 \mu \mathrm{T}$ at the coil center (the minus sign indicates the downward direction), which approximates the GMF in Sendai, Japan. In the active-sham mode, $B_{\mathrm{V}}=+7.4 \mu \mathrm{T}$ with the same amount of coil current. The AC magnetic field (up to $400 \mathrm{~Hz}$ ) was $<1 \mu \mathrm{T}$ in both coils. Hereafter, we designate the magnetic field for the exposure mode "-46 $\mu \mathrm{T}$ field" and that for the active-sham mode "+7.4 $\mu \mathrm{T}$ field". Flux densities in the exposure coil and the active-sham coil under various conditions are summarized in Table 1.

Table 1. DC magnetic fields in the Merritt coil

\begin{tabular}{cccccc}
\hline \multirow{5}{*}{ Exposure coil } & Coil & Incubator & $B_{\mathrm{V}}(\mu \mathrm{T})$ & $B_{\mathrm{H}}(\mu \mathrm{T})^{*}$ & $B(\mu \mathrm{T})^{* *}$ \\
\cline { 2 - 6 } & on & on & -46 & 17.6 & 49.3 \\
& off & on & +3.5 & 9.6 & 10.2 \\
\multirow{3}{*}{ Active-sham coil } & off & off & +1.6 & 9.6 & 9.7 \\
& on & on & +7.4 & 5.1 & 9.0 \\
$* B_{\mathrm{H}}$ represents the horizontal component of the magnetic flux density & & & 8.0 \\
$* * B=\sqrt{B \mathrm{~V}^{2}+B \mathrm{H}^{2}}$ & off & on & +6.3 & 5.0 & 4.6 \\
\hline
\end{tabular}

\subsection{Cell Culture}

Before the measurements, the macrophages were pre-cultured at $37^{\circ} \mathrm{C}$ in six or eight culture dishes $(\varnothing 6 \mathrm{~cm})$ containing cell culture medium (MEM supplemented with 1\% penicillin-streptomycin and 10\% heat-inactivated FBS) for 24-48 h in a $-46 \mu \mathrm{T}$ field to $20-50 \%$ confluency. The culture dishes were then split into two groups ( 3 or 4 dishes in each group), and each group was placed either in the Merritt coil system generating a $+7.4 \mu \mathrm{T}$ static field, or in a system generating a $-46 \mu \mathrm{T}$ field, for $24 \mathrm{~h}$ or $48 \mathrm{~h}$. The temperature in the incubators was recorded every $5 \mathrm{~min}$ with a data logger equipped with a thermistor probe (TR-71Ui; T \& D Corp., Matsumoto, Nagano, Japan) placed near the culture dishes. The time-average of the record indicated that the temperature was maintained at $37 \pm 0.1{ }^{\circ} \mathrm{C}$ during the pre-culture and experiment periods. Slight differences in the temperature of the exposure and active-sham coils were compensated for by adjusting the settings of the thermostat for each incubator, and the difference in temperature in the two incubators was kept $<0.2{ }^{\circ} \mathrm{C}$ throughout coil operation.

\subsection{Staining the Cells with JC-1}

Following exposure, cells suspended in MEM without phenol red (supplemented with non-essential amino acids and $10 \%$ FBS) were incubated at $37^{\circ} \mathrm{C}$ for 30 min with $5 \mu \mathrm{M} \mathrm{JC}-1$ (Polster et al., 2014). The fluorescence intensity of JC-1 was measured with a microplate reader (SH-9600 Lab; Corona Electric Japan, Hitachinaka, Japan) at $600 \mathrm{~nm}\left(I_{600}\right)$ and $540 \mathrm{~nm}\left(I_{540}\right)$ with excitation at $488 \mathrm{~nm}$ (Perelman et al., 2012).

\subsection{Measurement of the Number Density of the Cells}

The number density of the cells in each cell suspension $\left(\rho_{\text {cell }}\right)$ was determined in duplicate using an improved Neubauer counting chamber (Watson Bio Lab, Kobe, Japan).

\subsection{Measurement of $\mathrm{O}_{2}$}

The levels of $\mathrm{O}_{2}^{-}$in mitochondria were measured by staining the cells with MitoSox ${ }^{\mathrm{TM}}$ Red according to the manufacturer's instructions: after exposure, the cells were suspended in phosphate-buffered saline and labeled with 5 $\mu \mathrm{M}$ MitoSox ${ }^{\mathrm{TM}} \mathrm{Red}$ at $37^{\circ} \mathrm{C}$ for $1 \mathrm{~h}$. The fluorescence intensity was measured using the microplate reader at $580 \mathrm{~nm}$ $\left(I_{580}\right)$ with excitation at $510 \mathrm{~nm} . \mathrm{O}_{2}^{-}$levels in the cells were measured with nitro-TB according to the method of Choi et al. (2006). Cells were incubated with $0.1 \mathrm{mM}$ nitro-TB $+0.1 \mathrm{mM} \mathrm{CaCl}_{2}$ in MEM (no phenol red, no FBS, supplemented with non-essential amino acids) at $37{ }^{\circ} \mathrm{C}$ for $1 \mathrm{~h}$, then the cells were collected by centrifugation and suspended in phosphate-buffered saline. Part of the suspension was used to measure $\rho_{\text {cell }}$; the remaining cells were again pelleted. Blue formazan in the pellet, which was formed by the reaction between $\mathrm{O}_{2}^{-}$and nitro-TB, was dissolved in a 1:2 mixture of $2 \mathrm{M} \mathrm{KOH}$ and dimethylsulfoxide, and the absorbance was measured at $620 \mathrm{~nm}\left(A_{620}\right)$ using the microplate reader. 


\subsection{Staining Necrotic Cells With PI and Total Cells With Hoechst 33342}

Immediately following exposure, the cells were collected, suspended in phosphate-buffered saline that had been supplemented with $1 \%$ bovine serum albumin, and were divided into two parts. One part was mixed with propidium iodide to stain necrotic cells and the other part was mixed with Hoechst 33342 to stain the nuclei of all cells according to the manufacturer's instructions. The fluorescence intensity of propidium iodide $\left(I_{\mathrm{P}}\right)$ was measured at $535 \mathrm{~nm}$ with excitation at $617 \mathrm{~nm}$, and that of Hoechst $\left(I_{\mathrm{H}}\right)$ was measured at $440 \mathrm{~nm}$ with excitation at $357 \mathrm{~nm}$ using the microplate reader.

\subsection{Measurement of Cellular ATP}

Immediately after exposure, cellular ATP was measured according to the manufacturer's instructions. After collecting and suspending the cells in phosphate-buffered saline, the ATP assay reagent was added. The luminescence intensity of the mixture and that of phosphate-buffered saline alone were measured with the microplate reader. The difference between the two quantities was calculated and designated $I_{\mathrm{L}}$.

\subsection{Data Processing}

In each experiment, measurements of fluorescence intensity/absorbance were replicated three or four times, and the experiment under each condition was repeated several times. The fluorescence intensity of JC-1 was processed as follows. First, the emission ratios, $I_{600} / I_{540}$, obtained in triplicate measurements were averaged. The emission ratio reflects $\Delta \Phi_{\mathrm{m}}$ (Perelman et al., 2012). The emission ratios obtained from several independent experiments were further ensemble-averaged to determine the value of $\left\langle I_{600} / I_{540}\right\rangle$. We expressed the $\left\langle I_{600} / I_{540}\right\rangle$ value as $\left\langle E_{\text {ratio }}\right\rangle$. For measurements with MitoSox ${ }^{\mathrm{TM}}$ Red, the $I_{580}$ value for each microplate well was normalized to the total number of cells transferred to each well $\left(N_{\text {cell }}=\left[\rho_{\text {cell }}\right] \times 0.1 \mathrm{~mL}\right.$; the volume of the cell suspension in the well $)$. The ratios obtained from three wells were ensemble-averaged to determine the $I_{580} / N_{\text {cell }}$ value for that experiment. The values obtained from three independent experiments were ensemble-averaged ( $\left\langle I_{580} / N_{\text {cell }}>\right.$ value). For measurements with nitro-TB, the $A_{620}$ values were treated in the same manner as the $I_{580}$ values to determine the $\left\langle A_{620} / N_{\text {cell }}\right\rangle$ value. The level of cellular ATP was evaluated by normalizing the $I_{\mathrm{L}}$ values obtained from triplicate measurements to $N_{\text {cell }}\left(I_{\mathrm{L}} / N_{\text {cell }}\right)$. To evaluate the degree of necrosis, the $I_{\mathrm{P}}$ value obtained from triplicate measurements was normalized to $I_{\mathrm{H}}\left(I_{\mathrm{P}} / I_{\mathrm{H}}\right)$.

\subsection{Statistical Analyses}

The $\left\langle E_{\text {ratio }}\right\rangle,\left\langle I_{580} / N_{\text {cell }}>,\left\langle A_{620} / N_{\text {cell }}\right\rangle\right.$ and $\left\langle\rho_{\text {cell }}\right\rangle$ values were analyzed with two-tailed paired $t$-tests. The $I_{\mathrm{L}} / N_{\text {cell }}$ and $I_{\mathrm{P}} / I_{\mathrm{H}}$ values were subjected to Welch's test. Differences were considered statistically significant if $P<0.05$ and to show a tendency if $0.05<P<0.1$.

\section{Results}

\subsection{Effect of HMF Exposure on $\Delta \Phi_{m}$}

As shown in Figure 2, $\left\langle E_{\text {ratio }}\right\rangle$ for cells exposed to a $+7.4 \mu \mathrm{T}$ field for $24 \mathrm{~h}$ tended to be lower compared to cells exposed to a $-46 \mu \mathrm{T}$ field $(P=0.057 ; N=3)$, and this effect was more pronounced after $48 \mathrm{~h}$ exposure $(P=0.009 ; N=$ 3). In another set of experiments, exposure to a $+7.4 \mu \mathrm{T}$ field for $24 \mathrm{~h}$ did not significantly affect $\left\langle E_{\text {ratio }}\right\rangle(1.09 \pm 0.29$ for $-46 \mu \mathrm{T}$ vs. $1.08 \pm 0.32$ for $+7.4 \mu \mathrm{T} ; P=0.70, N=3)$, while $48 \mathrm{~h}$ exposure did significantly affect $\left\langle E_{\text {ratio }}\right\rangle(2.93 \pm$ 0.37 for a $-46 \mu$ T field vs. $2.83 \pm 0.31$ for a $+7.4 \mu \mathrm{T}$ field; $P=0.043, N=4$ ). Thus, the effect of the $+7.4 \mu \mathrm{T}$ field on $\left\langle E_{\text {ratio }}\right\rangle$ was apparent following a longer exposure period, suggesting that the $+7.4 \mu \mathrm{T}$ field lowered $\Delta \Phi_{\mathrm{m}}$.

We examined if rotenone, an inhibitor of Complex I in the electron transport chain in mitochondria, would decrease $\Delta \Phi_{\mathrm{m}}$, since a previous study demonstrated that rotenone decreases ATP production (Li et al., 2003), suggesting inhibition of mitochondrial function. We indeed observed that $1 \mu \mathrm{M}$ rotenone decreased $\left\langle E_{\text {ratio }}\right\rangle$ by $\sim 10 \%$. However, unlike rotenone, the $+7.4 \mu \mathrm{T}$ field did not affect cellular ATP, as shown in Table 2 . 


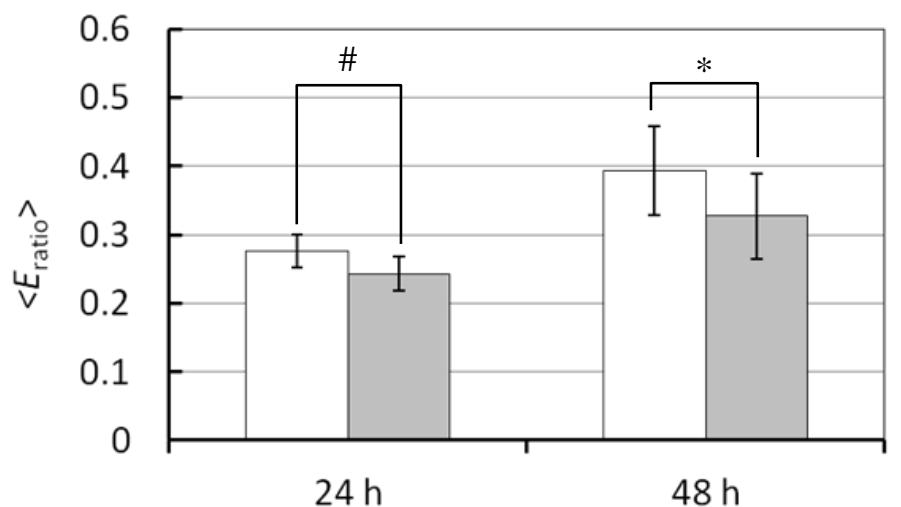

Figure 2. $\left\langle E_{\text {ratio }}>\right.$ values obtained after $24 \mathrm{~h}$ or $48 \mathrm{~h}$ exposure of macrophages to a $+7.4 \mu \mathrm{T}$ field. White bars, $-46 \mu \mathrm{T}$ field; grey bars, $+7.4 \mu \mathrm{T}$ field. Error bars, standard error of mean ( $N=3$ for both durations). The symbol \# represents $P$ $=0.057$, and $*$ represents $P=0.009$. This graph shows the results from the first set of experiments

Table 2. The effect of a $+7.4 \mu \mathrm{T}$ field on ATP production $\left(I_{\mathrm{L}} / N_{\text {cell }}\right)$ and necrosis $\left(I_{\mathrm{P}} / I_{\mathrm{H}}\right)$

\begin{tabular}{lcccccc}
\hline \multicolumn{3}{c}{ Experiment 1} \\
\hline & $+7.4 \mu \mathrm{T}$ & $-46 \mu \mathrm{T}$ & $P$ & $+7.4 \mu \mathrm{T}$ & $-46 \mu \mathrm{T}$ & $P$ \\
$I_{\mathrm{L}} / N_{\text {cell }}$ & $0.48 \pm 0.11$ & $0.55 \pm 0.11$ & 0.50 & $1.52 \pm 0.29$ & $1.57 \pm 0.32$ & 0.83 \\
$I_{\mathrm{P}} / I_{\mathrm{H}}$ & $0.038 \pm 0.005$ & $0.031 \pm 0.005$ & 0.12 & $0.035 \pm 0.004$ & $0.036 \pm 0.003$ & 0.64 \\
$*$ & & & & & \\
\hline
\end{tabular}

\section{$3.2 \mathrm{O}_{2}^{-}$Production}

We next examined if the $+7.4 \mu \mathrm{T}$ field increased $\mathrm{O}_{2}{ }^{-}$production, given that $\mathrm{Li}$ et al. (2003) demonstrated that rotenone induced a large ( three times) increase in $\mathrm{O}_{2}{ }^{-}$production. However, as shown in Figure $3 \mathrm{~A}$, the $\left\langle I_{580} / N_{\text {cell }}\right\rangle$ value for cells exposed to a $+7.4 \mu \mathrm{T}$ field for $24 \mathrm{~h}$ was not significantly different from that for cells exposed to a $-46 \mu \mathrm{T}$ field $(P=$ $0.25 ; N=3$ ); likewise, the $\left\langle I_{580} / N_{\text {cell }}>\right.$ value obtained using a $+7.4 \mu \mathrm{T}$ field was not significantly different from that obtained using a $-46 \mu \mathrm{T}$ field after $48 \mathrm{~h}$ exposure $(P=0.14 ; N=3)$. 
A
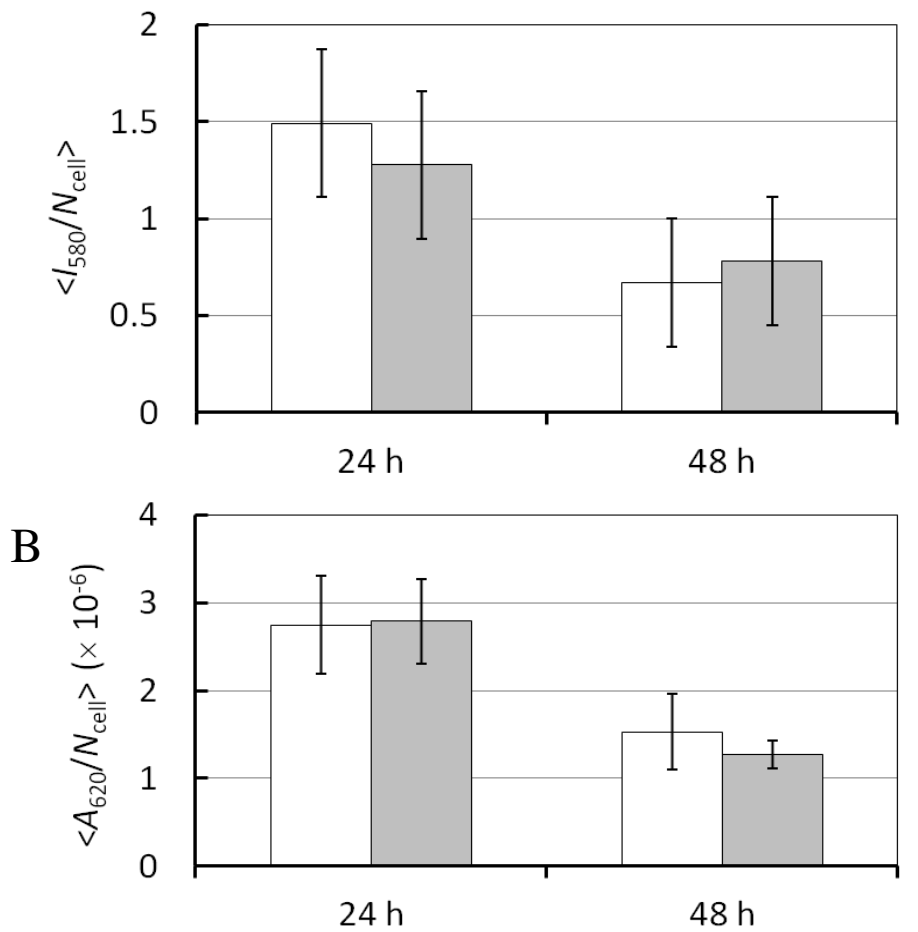

Figure 3. A) $\left\langle I_{580} / N_{\text {cell }}>\right.$ values for macrophages exposed to HMFs for $24 \mathrm{~h}$ or $48 \mathrm{~h}$ White bars, $-46 \mu \mathrm{T}$ field; grey bars, $+7.4 \mu \mathrm{T}$ field. Error bars, standard error of mean $\left(N=3\right.$ for both durations). B) $\left\langle A_{620} / N_{\text {cell }}\right\rangle$ values for macrophages exposed to an HMF for $24 \mathrm{~h}$ or $48 \mathrm{~h}$. White bars, $-46 \mu \mathrm{T}$ field; grey bars, $+7.4 \mu \mathrm{T}$ field. Error bars, standard error of mean $(N=3$ for both durations)

Figure 3B shows similar results obtained using the nitro-TB method: the $\left\langle A_{620} / N_{\text {cell }}\right\rangle$ value for cells exposed to a +7.4 $\mu \mathrm{T}$ field for $24 \mathrm{~h}$ was not significantly different from that for cells exposed to a $-46 \mu \mathrm{T}$ field $(P=0.83, N=3)$; the results following exposure for $48 \mathrm{~h}$ were similar $(P=0.55, N=3)$. Thus, the $+7.4 \mu \mathrm{T}$ field was not as effective as rotenone in affecting $\mathrm{O}_{2}^{-}$production, or it acted only on the mitochondrial membrane potential.

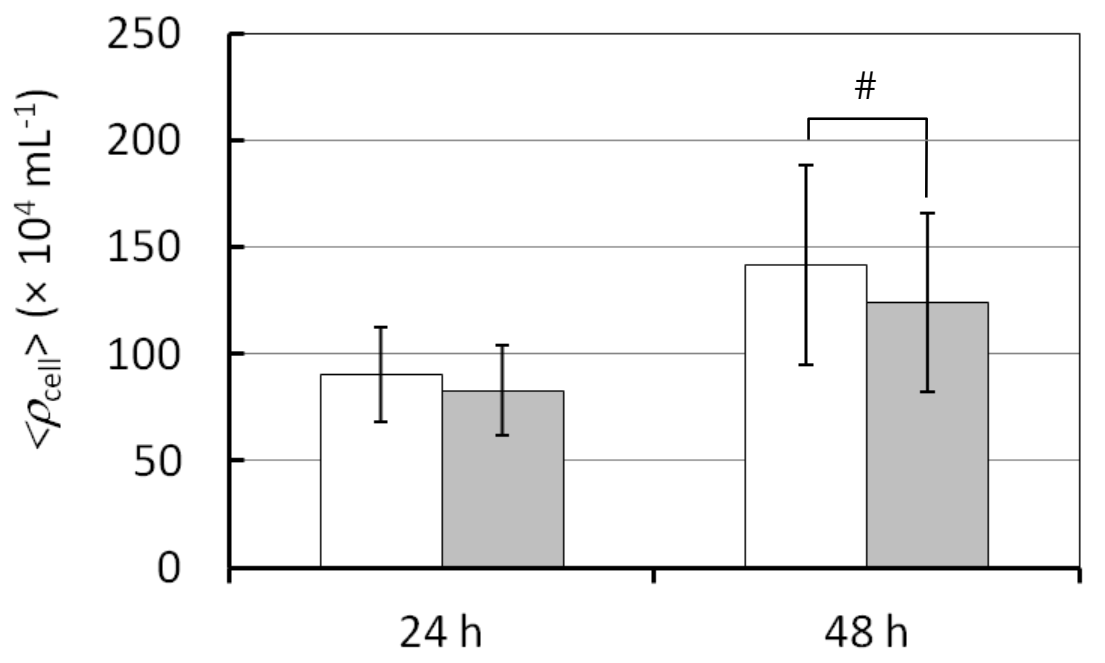

Figure 4. $\left\langle\rho_{\text {cell }}>\right.$ values for macrophages exposed to an HMF for $24 \mathrm{~h}$ or $48 \mathrm{~h}$. White bars, $-46 \mu \mathrm{T}$ field; grey bars, +7.4 $\mu \mathrm{T}$ field. Error bars, standard error of mean $(N=6$ for $24 \mathrm{~h} ; N=8$ for $48 \mathrm{~h} ; \#, P=0.06)$ 


\subsection{Effect of HMF Exposure on $\left\langle\rho_{\text {cell }}\right\rangle$}

As shown in Figure 4 , the $\left\langle\rho_{\text {cell }}>\right.$ value for cells exposed to a $+7.4 \mu \mathrm{T}$ field for $24 \mathrm{~h}$ was not significantly different from that for cells exposed to a $-46 \mu \mathrm{T}$ field $(P=0.23 ; N=6)$. $\left\langle\rho_{\text {cell }}\right\rangle$ tended to be lower when cells were exposed to a +7.4 $\mu \mathrm{T}$ field for $48 \mathrm{~h}(P=0.06 ; N=8)$.

We examined if the decrease in $\left\langle\rho_{\text {cell }}\right\rangle$ was due to increased necrosis. Two independent experiments demonstrated that the degree of necrosis, represented by $I_{\mathrm{P}} / I_{\mathrm{H}}$, for cells exposed to the $+7.4 \mu \mathrm{T}$ field was not significantly different from that for cells exposed to the $-46 \mu \mathrm{T}$ field (Table 2). This result is consistent with the result showing no difference in ATP level between cells exposed to the $+7.4 \mu \mathrm{T}$ field and the $-46 \mu \mathrm{T}$ field. Thus, it is unlikely that the $+7.4 \mu \mathrm{T}$ field decreased $\left\langle\rho_{\text {cell }}\right\rangle$ by promoting necrosis through ATP depletion (Zeiss, 2003).

\section{Discussion}

The present study demonstrated that $\Delta \Phi_{\mathrm{m}}$ significantly decreases $(\sim 17 \%)$ in cells exposed for $48 \mathrm{~h}$ to a $+7.4 \mu \mathrm{T}$ field. We reasoned that the $+7.4 \mu \mathrm{T}$ field affected $\Delta \Phi_{\mathrm{m}}$ in a similar manner to rotenone, which inhibits the Complex I in the electron transport chain in mitochondria, thereby increasing $\mathrm{O}_{2}^{-}$production (Turrens, 2003). And hence, we anticipated that the $+7.4 \mu \mathrm{T}$ field would increase $\mathrm{O}_{2}^{-}$production. However, the $\mathrm{O}_{2}^{-}$production was not significantly different between the $+7.4 \mu \mathrm{T}$ field and the $-46 \mu \mathrm{T}$ field. Thus, either the $+7.4 \mu \mathrm{T}$ field exerted a very weak effect on $\mathrm{O}_{2}{ }^{-}$ production, which we failed to detect, or it only acted on some process involved in $\Delta \Phi_{\mathrm{m}}$.

We also found that $\left\langle\rho_{\text {cell }}\right\rangle$ tended to decrease following exposure to the $+7.4 \mu \mathrm{T}$ field. This decrease was not due to an increase in necrosis through ATP depletion (Zeiss, 2003) because the $+7.4 \mu \mathrm{T}$ field did not lower the ATP level. One possible interpretation of the decrease in $\left\langle\rho_{\text {cell }}\right\rangle$ is an alteration in the cell cycle of the macrophages by the $+7.4 \mu \mathrm{T}$ field, as demonstrated with cancer cells exposed to HMFs (Martino et al., 2010).

A previous study (Fu et al., 2016b) showed that an HMF decreased $\Delta \Phi_{\mathrm{m}}$, ATP levels, and cell viability, and increased reactive oxygen species. The decrease in ATP level arising from the decreased mitochondrial function was previously assumed to cause the observed decrease in cell viability. The present study demonstrated that $\Delta \Phi_{\mathrm{m}}$ and $\rho_{\text {cell }}$ significantly decreased, but the $\mathrm{O}_{2}^{-}$level, the degree of necrosis, and the ATP level exhibited no significant changes. Fu et al. exposed skeletal muscle cells to a $<3 \mu \mathrm{T}$ HMF for three days, whereas in the present study, macrophages were exposed to a $7.4 \mu \mathrm{T}$ field for $48 \mathrm{~h}$. Apart from these obvious differences, we suggest that the $+7.4 \mu \mathrm{T}$ field might interfere with proton translocation across the inner mitochondrial membrane accompanying electron transport (Berg et al., 2003). We presume that this would only decrease $\Delta \Phi_{\mathrm{m}}$. Suppression of the electron transport process by the $7.4 \mu \mathrm{T}$ field could also decrease $\Delta \Phi_{\mathrm{m}}$, but we consider this is less likely as it would also elevate $\mathrm{O}_{2}^{-}$levels, similar to the inhibitory effect of rotenone on electron transport (Turrens, 2003). However, the present results show only a decrease in $\Delta \Phi_{\mathrm{m}}$.

The effect of the $\mathrm{HMF}$ on $\mathrm{O}_{2}{ }^{-}$production was weak, and thus faster and more sensitive analytical methods are needed to evaluate the effects of HMFs. Capillary electrophoresis (Lara et al., 2016) will fulfill this requirement. Thus, $\mathrm{O}_{2}{ }^{-}$in extracts of cells exposed to an HMF or the GMF could be separated from other chemical compounds before being detected using a fluorescence or chemiluminescence technique. $\mathrm{O}_{2}^{-}$production is regulated by a redox system in the cell, and altered $\mathrm{O}_{2}^{-}$levels would recover after exposure. The shorter analysis time of this technique (up to $10 \mathrm{~min}$, compared to $3 \mathrm{~h}$ with the present method) would be ideal for investigating the state of the cell immediately after exposure.

The mechanism by which the HMF lowered $\Delta \Phi_{\mathrm{m}}$ remains to be investigated. As described above, we speculate that the HMF interfered with proton translocation across the inner membrane that occurs during the electron transport process across protein complexes. Protons have a magnetic moment and thus can interact with magnetic fields and undergo precession. Precession in the GMF is regarded as a "natural background". Proton transport probably evolved in the presence of this background, and hence, the efficiency of this transport has been optimized for the GMF (Binhi and Prato, 2017a, b). If the external magnetic field is changed from the GMF to an HMF, conditions are no longer optimal and the efficiency of the reaction will be reduced. Another possibility is based on the radical pair mechanism, which depends on the interconversion of a radical pair between the singlet state and the triplet state (Lambert et al., 2013). Considering that a radical pair is generated in several metabolic processes, one may adopt this mechanism to explain the HMF effect (Zhang and Tian, 2020). Evaluating these two possibilities is a subject for future studies.

In this study, we cultured cells under an HMF by placing the $\mathrm{CO}_{2}$ incubator in a magnetic shielding case designed by the manufacturer to lower DC fields to $<1 \mu \mathrm{T}$. However, as shown in Table 1, a significant background DC magnetic field remained in the incubator even when the coil and incubator were turned off. This remnant field might be generated by the permanent magnets in the electric motor used to operate the circulating fan. The problem of background AC magnetic fields with spatial variation in incubators was previously pointed out (Mild et al., 2009). Non-uniform DC fields $(\sim 10 \mu \mathrm{T}$ to $\sim 100 \mu \mathrm{T})$ inside an incubator obtained from a different manufacturer resulted from the fan motor 
(Miyata, unpublished observation). The present study shows that a variation in the DC magnetic field as small as $50 \mu \mathrm{T}$ can alter experimental results and affect their interpretation in studies of small differences in physiological parameters. Every effort should thus be made to minimize the background DC field using proper shielding. Designing and constructing a "DC-field free" or "DC-field reduced" $\mathrm{CO}_{2}$ incubator and making it commercially available would resolve these problems.

The present study suggests that the effect of HMFs is rather weak but may cause adverse health effects after long-term exposure. It is also possible that the effect may be mitigated by homeostatic compensation in cells. Thus, the outcome by the HMF will be difficult to predict. This point should be further studied to elucidate the effect of HMFs on human health in space.

\section{Conclusion}

Cell exposure to an HMF lowered mitochondrial membrane potential and marginally decreased cell proliferation. We suggest that proton transport in mitochondria is the target of the HMF and this will be the subject of a future study. Whether the effect on mitochondria and cell proliferation is related remains an open question.

\section{Acknowledgments}

This work was supported by a Grant-in-Aid from MEXT, Japan (\#17K08563).

NS and WK were supported by the Internship Program (Overseas Co-operative Education Project by the Faculty of Science, Naresuan University; January 2018-May 2018 for NS and February 2019-April 2019 for WK). We are grateful to the Transportation Bureau of the City of Sendai for providing us with the two magnetic shield cases, and to Professor Tsuyoshi Hondou (Department of Physics, Tohoku University) for his valuable advices in the construction of the Merritt coil and magnetic shield case.

\section{References}

Belyavskaya, N. A. (2001). Ultrastructure and calcium balance in meristem cells of pea roots exposed to extremely low magnetic fields. Advances in Space Research, 28, 645-650. https://doi.org/10.1016/S0273-1177(01)00373-8

Belyavskaya, N. A. (2004). Biological effects due to weak magnetic field on plants. Advances in Space Research, 34, 1566-1574. https://doi.org/10.1016/j.asr.2004.01.021

Berg, J., Tymoczko, J., Stryer, L. (2003). Biochemistry. $5^{\text {th }}$ ed., Chapter 18. New York, NY: W.H. Freeman and Co.

Binhi, V. N., \& Prato, F. S. (2017a). Biological effects of the hypomagnetic field: an analytical review of experiments and theories. PLOS One, 12, 1-51. https://doi.org/10.1371/journal.pone.0179340

Binhi, V. N., \& Prato, F. S. (2017b). A physical mechanism of magnetoreception: extension and analysis. Bioelectromagnetics, 38, 41-52. https://doi.org/10.1002/bem.22011

Choi, H. S., Kim, J. W., Cha, Y. N. N., \& Kim, C. (2006). A quantitative nitroblue tetrazolium assay for determining intracellular superoxide anion production in phagocytic cells. Journal of Immunoassay Immunochemistry, 27, 31-44. https://doi.org/10.1080/15321810500403722

Fu, J. P., Mo, W. C., Liu, Y., \& He, R. Q. (2016b). Decline of cell viability and mitochondrial activity in mouse skeletal muscle cell in a hypomagnetic field. Bioelectromagnetics, 37, 212-222. https://doi.org/10.1002/bem.21968

Fu, J. P., Mo, W. C., Liu, Y., Bartlet, P. F., \& He, R. Q. (2016a). Elimination of the geomagnetic field stimulates the proliferation of mouse neural progenitor and stem cells. Protein \& Cell, 7, 624-637. https://doi.org/10.1007/s13238-016-0300-7

Gurfinkel, Y. I., At'kov, O.Y., Vasin, A. L., Breus, T. K., Sasonko, M. L., \& Pishchalinkov, R. Y. (2016). Effect of zero magnetic field on cardiovascular system and microcirculation. Life Sciences in Space Research, 8, 1-7. https://doi.org/10.1016/j.lssr.2015.11.001

Iorio, R., Delle Monache, S., Bennato, F., Di Bartolomeo, C., Scrimaglio, R., Cinque, B., \& Colonna, R. C. (2011). Involvement of mitochondrial activity in mediating ELF-EMF stimulatory effect on human sperm motility. Bioelectromagnetics, 32, 15-27. https://doi.org/10.1002/bem.20602

Kokhan, V. S., Matveeva, M. I., Mukhametov, A., \& Shtemberg, A. S. (2016). Risk of defeats in the central nervous system during deep space missions. Neuroscience \& Biobehavioral Reviews, 71, 621-632. https://doi.org/10.1016/j.neubiorev.2016.10.006

Lahijani, M. S., Tehrani, D. M., \& Fereydouni, N. (2013). Effects of $50 \mathrm{~Hz}$ extremely low frequency sinusoidal magnetic fields on the apoptosis of the hearts of preincubated chicken embryos at different levels of developments. International Journal of Radiation Biology, 89, 234-242. https://doi.org/10.3109/09553002.2013.754556 
Lambert, N., Chen, Y. N., Cheng, Y. C., Li, C. M., Chen, G. Y., \& Nori, F. (2013). Quantum biology. Nature Physics, 9 , 10-18. https://doi.org/10.1038/nphys2474

Lara, F. J., Airado-Rodríguez, D., Moreno-Gonzalez, D., Huertas-Perez, J., \& García-Campana, A. M. (2016). Applications of capillary electrophoresis with chemiluminescence detection in clinical, environmental and food analysis. A review. Analytica Chimica Acta, 913, 22-40. https://doi.org/10.1016/j.aca.2016.01.046

Li, N., Ragheb, K., Lawler, G., Sturgis, J., Rajwa, B., Melendez, J. A., \& Robinson, P. (2003). Mitochondrial complex I inhibitor rotenone induces apoptosis through enhancing mitochondrial reactive oxygen species production. Journal of Biological Chemistry, 278, 8516- 8525. https://doi.org/10.1074/jbc.M210432200

Martino, C. F., Portelli, L., McCabe, K., Hernandez, M., \& Barns, F. (2010). Reduction of earth's magnetic field inhibits growth rates of model cancer cell line. Bioelectromagnetics, 31, 649-655. https://doi.org/10.1002/bem.20606

Merritt, R., Purcell, C., \& Stroink, G. (1983). Uniform magnetic field produced by three, four, and five square coils. Review of Scientific Instruments, 54, 879-882. https://doi.org/10.1063/1.1137480

Mild, K. H., Wilen, J., Mattsson, M. O., \& Simko, M. (2009). Background ELF magnetic fields in incubators: A factor of importance in cell culture work. Cell Biology International, 33, 755-757. https://doi.org/10.1016/j.cellbi.2009.04.004

Perelman, A., Wachtel, C., Cohen, M., Haupt, S., Shapiro, H., \& Tzur, A. (2012). JC-1: alternative excitation wavelengths facilitate mitochondrial membrane potential cytometry. Cell Death \& Disease, 3, e430. https://doi.org/10.1038/cddis.2012.171

Polster, B. M., Nicholls, D. G., Ge, S. X., \& Roelofs, B. A. (2014). Use of Potentiometric Fluorophores in the Measurement of Mitochondrial Reactive Oxygen Species. Methods in Enzymology, 547, 225-250. https://doi.org/10.1016/B978-0-12-801415-8.00013-8

Pooam, M., Nakayama, M., Nishigaki, C., \& Miyata, H. (2017). Effect of 50-Hz sinusoidal magnetic field on the production of superoxide anion and the expression of heat-shock protein 70 in RAW264 cells. International Journal of Chemistry, 9, 23-36. https://doi.org/10.5539/ijc.v9n2p23

Roman, A., \& Tombarkiewicz, B. (2009). Prolonged weakening of the geomagnetic field (GMF) affects the immune system of rats. Bioelectromagnetics, 30, 21-28. https://doi.org/10.1002/bem.20435

Turrens, J. F. (2003). Mitochondrial formation of reactive oxygen species. J Physiol. 552(2), 335-344. https://doi.org/10.1113/jphysiol.2003.049478

Zeiss, C. J. (2003). The apoptosis-necrosis continuum: insights from genetically altered mice. Veterinary Pathology, 40, 481-495. https://doi.org/10.1354/vp.40-5-481

Zhang, B., \& Tian, L. (2020). Reactive oxygen species: potential regulatory molecules in response to hypomagnetic field exposure. Bioeletromagnetics. https://doi.org/10.1002/bem.22299

\section{Copyrights}

Copyright for this article is retained by the author(s), with first publication rights granted to the journal.

This is an open-access article distributed under the terms and conditions of the Creative Commons Attribution license (http://creativecommons.org/licenses/by/4.0/). 